\title{
Activités alimentaires et méryciques de moutons recevant des foins à l'auge
}

\author{
JP Dulphy, C Demarquilly
}

avec la collaboration de $M$ Dudilieu et $M$ Jailler

NRA, CRZV de Theix, station de recherches sur la nutrition des herbivores, I 63122 Saint-Genès-Champanelle, France

(Reçu le 12 décembre 1991; accepté le 2 mars 1992)

Résumé - Les quantités de matière sèche ingérées ainsi que les activités alimentaires et méryciques ont été mesurées simultanément pour 60 foins distribués à des moutons standard. Les animaux ont passé, en moyenne, $255 \pm 48 \mathrm{~min}$ à ingérer, $530 \pm 68 \mathrm{~min}$ à ruminer et ont ingéré $55,7 \mathrm{~g}$ de MS/kg P0.75 par j en $7,7( \pm 2.6)$ repas. Foins de graminées et foins de légumineuses ont été étudiés séparément. Lorsque la qualité du foin augmente (plus de matières azotées totales (MAT), moins de cellulose brute $(C B)$ ) la durée d'ingestion et le nombre de repas augmentent, la durée de rumination et la durée de mastication journalière diminuent. Dans le même temps les durées unitaires d'ingestion, de rumination et de mastication diminuent (durées/g de MS ingérée). Ces durées unitaires sont étroitement et négativement corrélées aux quantités ingérées. Les foins, distribués en jours courts, sont ingérés en plus faible quantité que les fourrages verts de départ, distribués en jours longs, pendant moins de temps et un peu plus rapidement. Par contre les durées de rumination sont identiques et la durée unitaire de mastication est plus élevée pour les foins.

ingestion / rumination / foin / ovin

Summary - Feeding and ruminating activities of sheep fed hay in the trough. Voluntary $d r y$ matter (DM) intake was measured together with feeding and ruminating activities for 60 hays (46 grasses and 14 legumes) fed to standard sheep. The animals spent an average of 255 min $(+48)$ eating, $530 \mathrm{~min}( \pm 68)$ ruminating and ingested $55.7 \mathrm{~g} \mathrm{DM} / \mathrm{kg}$ w.75 in $7.7(+2.6)$ meals each day (table l). When hay quality increased (more crude protein, less crude fiber) the time spent eating and the number of meals increased, and the time spent ruminating and the total time spent chewing each day decreased. Together, the unitary times (eating, ruminating and chewing per $g$ DM) decreased (tables II, III). These unitary times were strongly and negatively related to voluntary intake. The hays fed during short days (winter) are ingested in smaller quantities than the fresh green forage, fed during long days (spring), over a shorter time and more rapidly. However, the daily time spent ruminating is similar and the chewing time per $\mathrm{kg} D M$ is longer is for hays.

feed intake / rumination / hay / sheep 


\section{INTRODUCTION}

Les activités de mastication ont un rôle primordial dans la réduction des particules végétales afin qu'elles puissent passer du réseau vers le feuillet (Pearce et Moir, 1964; Ulyatt et al, 1986). La durée de ces activités et leurs facteurs de variation sont donc importants à connaître.

Nous avons entrepris, chez le mouton, un travail systématique sur ce sujet, les résultats concernant les fourrages verts (Dulphy et Demarquilly, 1974; Dulphy et Bechet, 1976; Dulphy et Michalet-Doreau, 1983) ont déjà été publiés, ainsi que ceux concernant les ensilages d'herbe (Dulphy, Michalet-Doreau et Demarquilly, 1984). Le travail présenté ici concerne les foins.

\section{MATÉRIEL ET MÉTHODES}

Soixante foins au total (14 de légumineuses et 46 de graminées) ont été étudiés au cours de plusieurs années. Tous avaient èté préparés dans de bonnes conditions, mais à des stades de récolte très différents.

Chacun de ces foins, après un hachage grossier dans un hache-paille (brins de $2-3$ $\mathrm{cm}$ ), a été distribué à volonté en 2 repas par jour pendant 3 semaines à un iot de 6 moutons (béliers castrés de race Texel âgés de 2-4 ans et pesant $45-75 \mathrm{~kg}$ ). Les activités alimentaires et méryciques des animaux ont été enregistrées au cours de la $3^{e}$ semaine selon la technique de Ruckebusch (1963).

Pendant cette même semaine les quantités de matière sèche (MS) ingérées étaient mesurées par différence entre les quantités offertes et les quantités refusées ( $10 \%$ de refus). La digestibilité de la matière organique (MO) a également été calculée après collecte totale des fèces.

Pour 38 de ces foins, les fourrages verts de départ avaient également été distribués à des moutons dans les conditions décrites par Dulphy et Bechet (1976). Les mêmes mesures avaient été faites.

Sur des échantillons représentatifs de chacun des fourrages offerts on a déterminé les te- neurs en matières azotées totales (MAT $=N \times$ 6,25; méthode de Kjeldahl) et en cellulose brute ( $C B$; méthode de Weende). Les teneurs en cendres (après incinération à $550^{\circ} \mathrm{C}$ ) ont été determinées sur les fourrages offerts et refusés ainsi que sur les fèces. Les teneurs en matière sèche ont été calculées après passage à l'étuve ventilée pendant $24 \mathrm{~h}$ à $80^{\circ} \mathrm{C}$.

Les définitions des diffèrents paramètres caractérisant les activités de mastication ont été données par Dulphy (1971) puis reprises par Dulphy et Demarquilly (1974). II s'agit des durées journalières d'ingestion, de rumination (en min/jour), des durées unitaires d'ingestion et de

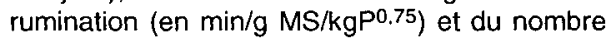
de repas. Nous appellerons mastication la somme des activités d'ingestion et de rumination.

\section{RÉSULTATS}

Les caractéristiques principales des foins étudiés figurent dans le tableau I. La diversité des foins utilisés a été importante puisque les valeurs extrêmes de leurs caractéristiques sont respectivement pour les MAT, 46-198 g/ $/ \mathrm{kg} \mathrm{MS}$, pour la cellulose brute $172-390 \mathrm{~g} / \mathrm{kg} \mathrm{MS}$ et pour la digestibilité $52-74,3 \%$. Pour les 60 foins, les moutons ont passé, en moyenne, $255( \pm 48$ ) min à ingérer et $530( \pm 68)$ min à ruminer. lis ont effectué $7,7( \pm 2,6)$ repas par jour. Entre foins de graminées et de légumineuses les durées de rumination sont légèrement différentes, mais compte tenu d'une ingestion plus élevée pour ces derniers les durées unitaires d'ingestion et de rumination sont nettement plus faibles pour les foins de légumineuses.

C'est la durée journalière de mastication qui est le paramètre le moins variable pour les foins de graminées ( $\mathrm{CV}$ de 10\%) et la durée journalière de rumination ( $C V$ de $12 \%$ ) pour chaque catégorie de foin. Les autres paramètres du comportement sont beaucoup plus variables, surtout le nombre de repas par jour ( $C V$ de $31 \%$ ). 
Tableau I. Caractéristiques des foins et valeurs des paramètres du comportement alimentaire et mérycique (moyenne, écart type).

\begin{tabular}{|c|c|c|c|}
\hline & $\begin{array}{l}\text { Graminées } \\
\quad \mathrm{n}=46\end{array}$ & $\begin{array}{l}\text { Légumineuses } \\
\qquad n=14\end{array}$ & \\
\hline \multicolumn{4}{|l|}{ Teneur en $\mathrm{g} / \mathrm{kg} M S$ : } \\
\hline Matières azotées totales (MAT) & $109 \pm 32$ & $166 \pm 20$ & $\mathrm{~s}$ \\
\hline Cellulose brute $(C B)$ & $296 \pm 41$ & $292 \pm 60$ & NS \\
\hline Digestibilité de la MO (\%) & $63,9 \pm 5,2$ & $61,5 \pm 5,2$ & NS \\
\hline Quantités de MS ingérées ( $\left.\mathrm{g} / \mathrm{kgP}^{0,75}\right)$ & $52,7 \pm 9,8$ & $65,7 \pm 11,9$ & $\mathrm{~s}$ \\
\hline \multicolumn{4}{|l|}{ Durée en $\min / j$ : } \\
\hline Ingestion (DI) & $256 \pm 43$ & $250 \pm 65$ & NS \\
\hline Rumination (DR) & $541 \pm 67$ & $496 \pm 58,5$ & $S$ \\
\hline Mastication (DM) & $797 \pm 79$ & $746 \pm 117$ & NS \\
\hline \multicolumn{4}{|l|}{ Durées unitaires $\mathrm{min} / \mathrm{g} M S / \mathrm{kg} ; P^{0,75}$} \\
\hline Ingestion (DUI) & $4,95 \pm 0,93$ & $3,88 \pm 0,97$ & $S$ \\
\hline Rumination (DUR) & $10,65 \pm 2,57$ & $7,82 \pm 1,86$ & $\mathrm{~s}$ \\
\hline Mastication (DUM) & $15,60 \pm 3,13$ & $11,71 \pm 2,66$ & $S$ \\
\hline Nombre de repas $(\geq 7 \mathrm{~min} / \mathrm{j})$ & $7,7 \pm 2,4$ & $7,7 \pm 2,3$ & NS \\
\hline
\end{tabular}

Les relations linéaires entre les principales caractéristiques des foins et les paramètres du comportement alimentaire figurent dans le tableau II lorsqu'elles sont significatives. Les teneurs en MAT et $C B$ jouent un rôle important, dans un sens toujours opposé, pour expliquer la variabilité des paramètres du comportement. Ainsi la durée d'ingestion augmente avec la teneur en $M A T$, de même que le nombre de repas par jour. La durée de rumination diminue lorsque la teneur en MAT augmente, mais augmente avec la teneur en cellulose brute. Il en est de même pour la durée de mastication. L'influence respective de ces 2 critères de la qualité des foins est cependant différente selon les paramètres du comportement considérés. La durée unitaire d'ingestion dépend par exemple plus étroitement de la teneur en $C B$ avec laquelle elle est positivement liée. C'est l'inverse pour la durée unitaire de rumination, surtout liée, et négativement, à la teneur en MAT. De même la durée unitaire de mastication est plus liée à la teneur en $M A T$, et négativement. Enfin le nombre de repas diminue lorsque la teneur en $C B$ du foin augmente.

La prise en compte simultanée des teneurs en $M A T$ et $C B$ des foins améliore les relations présentées ci-dessus, et surtout les durées unitaires d'ingestion, de rumination et de mastication (tableau III). Par contre la digestibilité de la MO n'influence significativement et négativement, comme les MAT, que les durées unitaires, mais de façon négligeable par rapport aux 2 critères retenus; nous n'avons donc pas donné les relations correspondantes.

Lorsque l'ingestibilité ( $Q I)$ des foins augmente (tableau II), la durée de mastication demeure constante, par suite d'une augmentation légère, mais significative de la durée d'ingestion et d'une baisse, elle- 
Tableau II. Influence des principales caractéristiques des foins sur les paramètres du comportement alimentaire (relations significatives seuiement de type $y=a+b x$ ).

\begin{tabular}{|c|c|c|c|c|c|c|}
\hline $\begin{array}{l}\text { Caractéristique } \\
\text { du fourrage }(x)\end{array}$ & $\begin{array}{l}\text { Paramètre } \\
(y)\end{array}$ & $\begin{array}{c}G: \text { graminées } \\
T: \text { tous les foins }\end{array}$ & a & $b$ & $\begin{array}{l}\text { Écart type } \\
\text { Syx }\end{array}$ & $r$ \\
\hline \multirow[t]{11}{*}{ MAT } & $D I$ & $\mathrm{G}$ & 197 & $+0,543$ & 39 & 0,41 \\
\hline & $D R$ & G & 662 & $-1,107$ & 57 & $-0,53$ \\
\hline & & $\mathrm{T}$ & 652 & $-0,990$ & 56 & $-0,56$ \\
\hline & $D M$ & $T$ & 875 & $-0,734$ & 87 & $-0,31$ \\
\hline & $D U I$ & $G$ & 5,87 & $-0,009$ & 0,89 & $-0,30$ \\
\hline & & $T$ & 6,33 & $-0,013$ & 0,90 & $-0,50$ \\
\hline & DUR & $\mathrm{G}$ & 17,28 & $-0,060$ & 1,67 & $-0,77$ \\
\hline & & $T$ & 16,94 & $-0,057$ & 1,59 & $-0,81$ \\
\hline & DUM & $\mathrm{G}$ & 23,16 & $-0,069$ & 2,22 & $-0,71$ \\
\hline & & $T$ & 23,27 & $-0,070$ & 2,15 & $-0,78$ \\
\hline & Repas & $\mathrm{G}$ & 5,08 & $+0,024$ & 2,28 & 0,33 \\
\hline \multirow[t]{11}{*}{$\mathrm{CB}$} & $D R$ & $\mathrm{G}$ & 359 & $+0,615$ & 63 & 0,37 \\
\hline & & $T$ & 347 & $+0,621$ & 62 & 0,42 \\
\hline & $D M$ & $\mathrm{~T}$ & 548 & $+0,804$ & 84 & 0,40 \\
\hline & DUI & G & 1,50 & $+0,012$ & 0,81 & 0,51 \\
\hline & & $\mathrm{T}$ & 0,99 & $+0,013$ & 0,87 & 0,55 \\
\hline & DUR & G & $-1,1$ & $+0,039$ & 2,03 & 0,62 \\
\hline & & $T$ & 0,64 & $+0,032$ & 2,30 & 0,53 \\
\hline & DUM & $\mathrm{G}$ & 0,49 & $+0,051$ & 2,38 & 0,66 \\
\hline & & $\mathrm{T}$ & 1,62 & $+0,044$ & 2,81 & 0,58 \\
\hline & Repas & G & 16,3 & $-0,029$ & 2,06 & $-0,52$ \\
\hline & & $\mathrm{T}$ & 13,7 & $-0,020$ & 2,1 & $-0,41$ \\
\hline \multirow[t]{9}{*}{ QI } & $D I$ & $G$ & 140 & $+1,203$ & 37 & 0,51 \\
\hline & & $T$ & 174 & $+1,433$ & 46 & 0,35 \\
\hline & DUI & $\mathrm{G}$ & 7,73 & $-0,053$ & 0,78 & $-0,56$ \\
\hline & & $T$ & 7,84 & $-0,056$ & 0,81 & $-0,63$ \\
\hline & DUR & $\mathrm{G}$ & 22,32 & $-0,221$ & 1,38 & $-0,85$ \\
\hline & & $\mathrm{T}$ & 21,21 & $-0,201$ & 1,34 & $-0,87$ \\
\hline & DUM & G & 30,05 & $-0,274$ & 1,63 & $-0,86$ \\
\hline & & $\mathrm{T}$ & 29,05 & $-0,257$ & 1,70 & $-0,87$ \\
\hline & Repas & G & 0,91 & $+0,129$ & 2,01 & 0,55 \\
\hline
\end{tabular}

Durée en $\mathrm{min} / \mathrm{j}=D I:$ ingestion; $D R:$ rumination; $D M:$ mastication. Durées unitaires min $/ \mathrm{g} \mathrm{MS} / \mathrm{kg}: P^{0.75}=D U I$ : ingestion; DUR: rumination; DUM: mastication.

même légère, mais non significative, de la durée de rumination. Dans ces conditions les durées unitaires sont étroitement et négativement corrélées aux quantités ingérées.

Les comparaisons effectuées entre fourrages verts et foins figurent dans le tableau IV. Les foins sont ingérés en plus faible quantité $(83 \%$ des fourrages verts), pendant moins de temps et un peu plus rapidement (ingestion (DUI) plus faible) que les fourrages verts. Par contre les durées de rumination sont identiques, ce qui signifie que chaque gramme de foin 
Tableau III. Influence combinée des teneurs en MAT et CB sur les caractéristiques du comportement alimentaire (relations amélioratrices par rapport à celles du tableau II).

\begin{tabular}{|c|c|c|c|c|}
\hline \multicolumn{3}{|c|}{$\begin{array}{c}G: \text { graminées } \\
T: \text { tous les foins }\end{array}$} & \multirow{2}{*}{$\begin{array}{c}\text { Syx } \\
53,7\end{array}$} & \multirow{2}{*}{$\begin{array}{c}R \\
0,61\end{array}$} \\
\hline$D R(\mathrm{~min} / \mathrm{j})$ & $\mathrm{T}$ & $526-0,817 M A T+0,346 C B$ & & \\
\hline$D U /\left(\mathrm{min} / \mathrm{g}\right.$ par kg, $\left.\mathrm{P}^{0,75}\right)$ & $\mathrm{T}$ & $3,15-0,0097 M A T+0,0094 C B$ & 0,82 & 0,65 \\
\hline$D U R\left(\min / g\right.$ par kg, $\left.\mathrm{P}^{0,75}\right)$ & $\begin{array}{l}\mathrm{G} \\
\mathrm{T}\end{array}$ & $\begin{array}{l}10,52-0.0482 M A T+0,018 C B \\
12.01-0.510 M A T+0,0296 C B\end{array}$ & $\begin{array}{l}1,57 \\
1,47\end{array}$ & $\begin{array}{l}0,80 \\
0,85\end{array}$ \\
\hline$D U M\left(\min / g\right.$ par kg, $\left.P^{0,75}\right)$ & $\stackrel{G}{T}$ & $\begin{array}{l}12,16-0,049 M A T+0,0296 C B \\
15,16-0,061 M A T+0,0240 C B\end{array}$ & $\begin{array}{l}2,00 \\
1,88\end{array}$ & $\begin{array}{l}0,78 \\
0,85\end{array}$ \\
\hline
\end{tabular}

Pour abréviations, voir tableau II.

Tableau IV. Comparaison des paramètres du comportement alimentaire entre foins et fourrages verts initiaux $(n=38)$.

Foins Fourrages verts

Quantités de MS ingérées $(g / k g, P 0.75)$

54,5

65,5

$S$

Durées en $\min / j$ :

Ingestion

239

518

Rumination

757

318

516

834

NS

Mastication

Ingestion

14,47

NS

Nombre de repas par jour

9,4

10,0

NS

est plus mastiquée lors de la rumination. Compte tenu de ces résultats la durée de mastication des foins est plus faible que celle des fourrages verts, mais la durée unitaire de mastication est plus élevée.

\section{DISCUSSION ET CONCLUSION}

II n'existe pas, à notre connaissance, d'études systématiques publiées sur les activités alimentaires et méryciques de moutons recevant des foins. 
II est intéressant de noter cependant que la durée journalière de mastication ne varie pas avec les quantités de fourrage ingérées. Nous avions trouvé pratiquement le même résultat pour des fourrages verts de graminées (Dulphy et Demarquilly, 1974), avec une constatation presque identique : lorsque l'ingestion augmente la durée journalière d'ingestion a tendance à augmenter et celle de rumination à diminuer.

Par contre la durée journalière de mastication augmente lorsque la teneur en MAT du fourrage diminue (environ 100 min entre les extrêmes) et surtout lorsque la teneur en $C B$ augmente (environ 170 min entre les extrêmes), ce qui indique que lorsque la qualité du fourrage diminue les animaux passent plus de temps à mastiquer, surtout lors de la rumination. Comme pour les fourrages verts (Dulphy et Bechet, 1976) la durée moyenne maximale de rumination atteinte par les moutons est de l'ordre de 600 min par jour.

Dans la mesure où les variations des durées d'ingestion et de rumination ne sont pas considerables on observe par contre des variations très nettes des durées unitaires avec les caractéristiques des foins et leur niveau d'ingestion. Ainsi, lorsque la qualité du fourrage diminue, il faut plus de temps aux animaux pour ingérer et ruminer une même quantité. Ceci est classique (Balch, 1971; Dulphy et Bechet, 1976). On parle alors d'une augmentation de l'indice de fibrosité du fourrage (Sauvant et al, 1990). Ce indice, exprimé en durée de mastication par kg de MS augmente donc lorsque la teneur en MAT diminue, alors que la teneur en $C B$ augmente, traduisant l'augmentation de la résistance du fourrage à être divisé en fines particules.

La durée unitaire de mastication des foins est légèrement plus élevée que celle des fourrages verts, ce qui est logique compte tenu de l'augmentation de leur teneur en $C B$ et de la baisse de leur teneur en MAT par rapport aux fourrages verts initiaux (Andrieu et al, 1981).

Notons enfin que les durées unitaires de mastication et les quantités ingérées sont étroitement corrélées :

$$
D U M=29,05-0,257 Q I \pm 1,70(r=-0,87)
$$

Nous avions trouvé une relation donnant des valeurs tout à fait comparables pour les fourrages verts (Dulphy et Bechet, 1976) : $D U M=26,68-0,207$ Q $(r=-0,92)$.

En conclusion, cette étude complète et valide nos études systématiques antérieures sur différents types de fourrages. Elles peuvent servir, au même titre que les études sur la vitesse de digestion des fourrages ou sur le niveau de réplétion du rumen, à comprendre les mécanismes impliqués dans le contrôle des quantités de fourrage ingérées chez le ruminant (Baumont, 1989).

\section{RÉFÉRENCES}

Andrieu J, Demarquilly C, Wegat-Litre E, Weiss $P(1981)$ Prévision de la valeur énergétique des foins. In : Prévision de la valeur nutritive des aliments des ruminants (C Demarquilly, ed) INRA Publ, 119-127

Balch CC (1971) Proposal to use time spent chewing as an index of the extent to which diets for ruminants possess the physical property of fibrousness characteristic of roughages. Br J Nutr 26, 383-392

Baumont $R$ (1989) État de répletion du réticulorumen et ingestion de fourrages : incidences sur le contrôle à court terme de la quantité de foin ingérée par le mouton. Thèse INRA Paris-Grignon, $159 \mathrm{p}$

Dulphy JP (1971) Influence du poids vif et du niveau d'ingestion sur le comportement alimentaire et mérycique du mouton. Ann Zootech 20, 477-486

Dulphy JP, Demarquilly C (1974) Étude du comportement alimentaire et mérycique de moutons recevant des fourrages verts hachés. Ann Zootech 23, 193-212 
Dulphy JP, Bechet G (1976) Influence du stade de végétation et de l'espèce végétale sur le comportement alimentaire et mérycique de moutons recevant des fourrages verts hachés. Ann Zootech 25, 505-519

Dulphy JP, Michalet-Doreau B (1983) Comportement alimentaire et mérycique d'ovins et de bovins recevant des fourrages verts. Ann Zootech 32, 465-474

Dulphy JP, Michalet-Doreau B, Demarquilly C (1984) Étude comparée des quantités ingérées et du comportement alimentaire et mérycique d'ovins et de bovins recevant des ensilages d'herbe réalisés selon différentes techniques. Ann Zootech 33, 291-320

Pearce GR, Moir RJ (1964) Rumination in sheep. I. The influence of rumination and grinding upon the passage and digestion of food. Aust J Agric Res 15, 635-644

Ruckebusch Y (1963) Recherches sur la régulation centrale du comportement alimentaire chez les ruminants. Thèse université de Lyon, $213 p$

Sauvant D, Dulphy JP, Michalet-Doreau B (1990) Le concept d'indice de fibrosité des aliments des ruminants. INRA Prod Anim 3, 309-318

Ulyatt MJ, Dellow DW, John A, Reid CSW, Waghorn GC (1986) Contribution of chewing during eating and rumination to the clearance of digesta from the reticulo-rumen. In: Control of digestion and metabolism in ruminants (LP Milligan, WL Grovum, A Dobson, eds) Prentice Hall, Englewood Cliffs, New Jersey, 498-515 